\title{
Qualification of CT data for areal surface texture analysis
}

\author{
Yann Quinsat • Jean Baptiste Guyon • \\ Claire Lartigue
}

Received: date / Accepted: date

\begin{abstract}
The evolution of current manufacturing processes, such as additive manufacturing processes, enables to produce parts with increasingly complex internal and external geometries, to answer functional requirements. This requires an evolution of the measurement methods to analyze the complete part produced. In this context, the use of computed tomography (CT) is increasing. Considering the problem of surface quality control, and also considering the cost of such a measuring system, it becomes necessary to evaluate the capability of tomography techniques to characterize surface geometry despite an inadequate resolution. To this end, the study proposed in this paper aims at assessing the quality of surface roughness characterization by $\mathrm{CT}$ in comparison with classical optical measure means. Special attention is given to thresholding, which isnecessary to extract the surface from CT measurements, which are the basis to evaluate roughness parameters. An advanced analysis is also performed to bring out surface typologies that are more appropriate for CT measurements with poor resolution.
\end{abstract}

Keywords CT measurement · Threshold · Surface Extraction · Surface Topography

\section{Introduction}

The 3D topography of manufactured surfaces is a critical factor in relation with the associated part function. Indeed, the external surface of a part is the interface between various physical, optical and mechanical phenomena. For instance, reflection in optics or friction in tribology are physical phenomena which strongly depend on the roughness of the external part surface, which itself strongly depends on process parameters. As a result, the relations between manufacturing process parameters and the functionality of the part are numerous and complex. From

Y. Quinsat

LURPA, ENS Paris-Saclay, Univ. Paris-Sud, Université Paris-Saclay, 94235 Cachan, France Tel.: +33147402213

Fax: +33147402220

E-mail: yann.quinsat@ens-paris-saclay.fr 
a standardization point of view, the characterization of surface roughness can be carried out in three dimensions [6], thus reflecting the anisotropic aspect of the surface.

Nowadays, manufacturing processes rapidly evolve. Within this context, additive manufacturing allows the elaboration of complex geometries, which are optimized in order to satisfy mechanical or physical performance characteristics. These structures, such as lattice structures [2], present both internal and external 3D topographies, which fully participates to the overall mechanical properties of the part. To characterize the overall surface topography, measurement methods have evolved to Computed Tomography (CT) $[4,27,22]$. In previous works $[18,19]$, we have shown that both the internal and external geometry can be characterized using CT measurements, and afterwards can be linked to process parameters. However, despite the improvement of CT measurement quality, it still remains difficult to measure mechanical parts having classical dimensions with a sufficient resolution to study 3D surface texture (or roughness). This problem appears when measuring parts with large dimensions that do not allow the part to be positioned close enough to the X-ray source (figure 1).

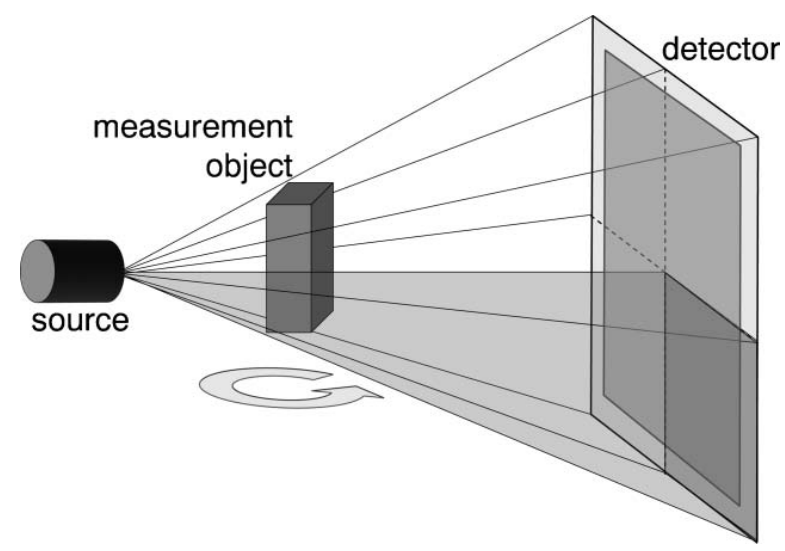

Fig. 1 Principle of Computed Tomography measurement [27]

The originality of this paper lies in the assessment of the ability of CT measurements to characterize surface texture despite an inadequate resolution.

\subsection{Related works}

Only a few studies have addressed the issue of measuring the surface roughness on classical size parts using computed tomography. Aloisi and Carmignato [1] studied and characterized the influence of surface roughness on X-ray CT dimensional measurements. They have pointed out that surface roughness causes a systematic error between classical touch probe measurements and CT measurements, but their study does not propose a direct qualification of CT measurements for 3D surface topography evaluation. Diaz et al. [5] proposed to evaluate texture parameters, 
such as skewness, kurtosis, and maximum surface height, from X-CT measurements of large rock blocks. However, their study was limited to the roughness of open and closed joints in rock, and not on high-density materials that compose mechanical parts.

Literature is more extensive when it comes to studying smaller specimens. These specimens allow a better resolution of the measuring system. Pyka et al. [17] used micro-CT for evaluating surface roughness of Ti6Al4V lattice structures obtained by Selective Laser Melting (SLM). In this study, the authors show that post additive manufacturing surface treatment can be optimized by applying a design of experiments using CT measurements. Kerckhofs et al. [8] showed that micro-CT can be accurately and robustly used for surface roughness characterization. This statement relies on a comparative study performed between texture parameters obtained using a commercial measuring system combining optical and touch probes, and CT-based roughness measurements with a resolution of $1.3 \mu \mathrm{m}$ (corresponding to the voxel size). Townsend et al. [25] extracted surface information from XCT volume data (with a $17.3 \mu \mathrm{m}$ voxel size) using VGStudio MAX 2., which supports the calculation of surface texture parameters. The specimen used is an additive manufactured part from AlSi10Mg aluminium alloy powder. The authors showed that parameters calculated that way are similar to those obtained when using conventional optical measuring techniques dedicated to surface roughness measurements. Nevertheless, the limited resolution of CT measurement is identified as the primary obstacle preventing the use of CT for surface topography measurements of additive manufacturing parts [21]. Using several measuring systems including CT, Senin et al. [20] show that no single measurement technology provides a completely reliable rendition of the topographic features to characterise the metal powdered fusion process.

One of the difficulties of the surface topography analysis from volumetric data is linked to data segmentation and part surface extraction. Moroni and Petró [13] clearly showed the influence of the threshold choice on dimensional measurement results. They conluded that specific guideline need to be developed for the choice of the threshold value. Townsend et al. [23] explained that CT could be useful for surface texture measurements in additive manufacturing, and also showed [26] that the threshold choice has a significant impact on the extracted surface texture. Although analyses from CT are close enough from optical measurements to assess the process accurately, thresholds are determined by a local iterative operation defined in the VGStudio MAX 2.2 software.

\subsection{Surface texture analysis from CT measurements}

The whole process for data analysis is outlined in Figure 2. The first step is the creation of a .raw file during the CT measurement. To assess the ability of CT measurements to characterize surface texture, our approach proposes to use various thresholds for surface extraction. To this purpose, after importing the stack of images into MatLab, a region of interest is selected by the user on one representative image (commonly in the middle of the stack).

Three thresholds, that will be presented in Section 2.1, are estimated from the image histogram of grey levels. The complete stack is afterwards processed to separate the part from the background and an envelope is created. This step, 


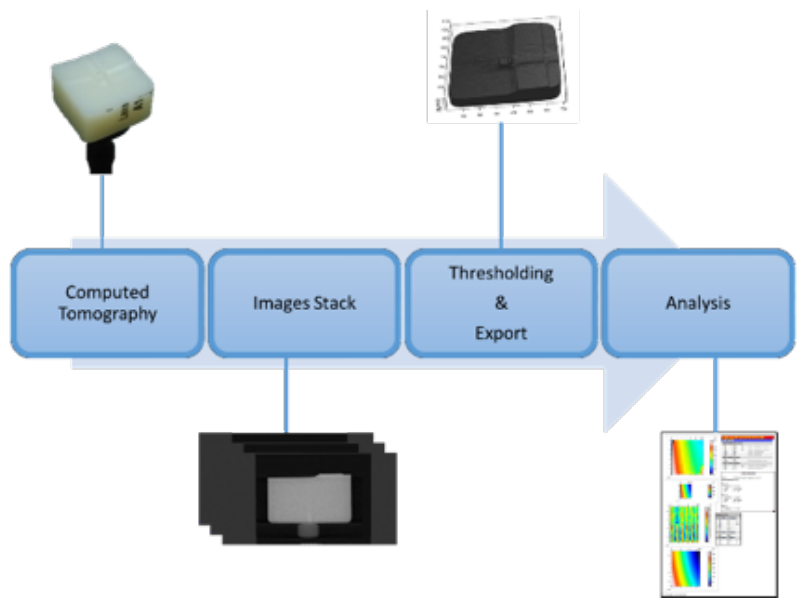

Fig. 2 Description of data analysis process

Table 1 Surface Texture Parameters [6]

\begin{tabular}{lll}
\hline Parameter & Unit & Name \\
\hline$S_{a}$ & $\mu m$ & Arithmetical mean deviation \\
$S_{q}$ & $\mu m$ & Root mean squared \\
$S_{z}$ & $\mu m$ & Maximum height of the surface \\
$S_{v}$ & $\mu m$ & Maximum height of valleys \\
$S_{p}$ & $\mu m$ & Maximum height of peaks \\
$S_{k u}$ & No unit & Kurtosis of height distribution \\
$S_{s k}$ & No unit & Skewness of height distribution \\
$S_{d q}$ & No unit & Root mean square gradient of the sur- \\
& & face \\
$S_{d r}$ & No unit & Developed area ratio \\
$S_{a l}$ & $\mu m$ & Fastest decay auto-correlation rate \\
$S_{5 v}$ & $\mu m$ & 5 point valley height
\end{tabular}

described in Section 2, leads to the extraction of the part envelope as a set of points that are then recovered in a .txt file, which is the ready to be exported and analysed using different types of software. The last steps are performed using MountainMap software for surface analyses. The same procedure is used for the analysis of different part areas. A region of interest is selected, and it is then filtered using a morphological filter to remove aberrant points. The so filtered region is the basis for parameter estimation. Those surface texture parameters issued from the standard [6] are described in Table 1.

\section{Surface extraction based on thresholding}

\subsection{Thresholding description}

The thresholding operation is an important step in the treatment of the volumetric data obtained with CT measurements, as it yields to the part surface. Classically, 


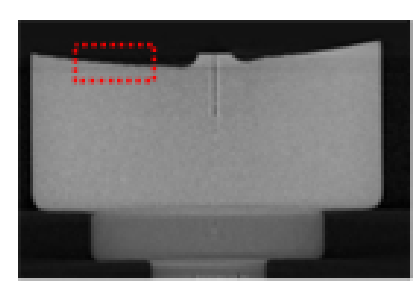

Fig. 3 Exemple of a slice of the volumetric data

the value of the threshold is defined from the grayscale histogram of the volumetric data. Most of the time the grayscale distribution ranges from black, which corresponds to air, to lighter values corresponding to the measured part.

\subsubsection{Threshold determination}

The threshold has to be equal to the brightness of the darkest pixel in the sample in order to remove the pixels representing the environment, and keep all the pixels of the object. This value is difficult to obtain because of the heterogeneity of the material of the sample, and because of the blur caused by the use of Xrays. However, it is possible to approach this value using the grayscale histogram. The simplest way to define the threshold value is to compute the $I S O_{50} T_{I_{s 0_{50}}}$ threshold (see section 2.1.2). But one main difficulty lies in the selection of a global threshold considering local variations. Indeed, a unique value for the entire model only effectively works if the workpiece is uniform, and homogeneous. Kowaluk and Wozniak [9] suggested improving the $I S O_{50}$ threshold using additional measurements with calibrated workpieces to determine corrections to be applied to the previous determined threshold. Obrist et al. [15] applied a different threshold value to each image, corresponding to a slice of the CT image stack. Torabella et al. [24] proposed a method based on a 3D Canny algorithm rather than the threshold technics, and show it is well adated for complexe surface extraction.

In this paper, only a portion of the volumetric data corresponding to the studied surface region is analysed. This allows us to consider the threshold choice as a local problem only. To illustrate our purpose, we use the image proposed in Figure 3. The corresponding histogram of gray levels is displayed in Figure 4. Due to the resolution of $\mathrm{CT}$ measurements that could be inadequate, it is essential to first assess the choice of an adapted threshold. To this aim, we propose to calculate the threshold value using three different methods, and to study the influence of each on the areal texture parameters.

\subsubsection{ISO50 Threshold}

The $I S O_{50}$ threshold, $T_{I_{s o_{50}}}$, is defined by the histogram of all gray values represented in the volumetric model. The peak corresponding to the object material is set to $100 \%$ and the other peak, which corresponds to air noise, is set to $0 \%$. The value in the middle of these two peaks, which corresponds to $50 \%$, defines the $I_{S} O_{50}$ threshold used for surface extraction. Using this calculation method, the $I_{S} O_{50}$ threshold corresponds to the red vertical line in Figure 4. 


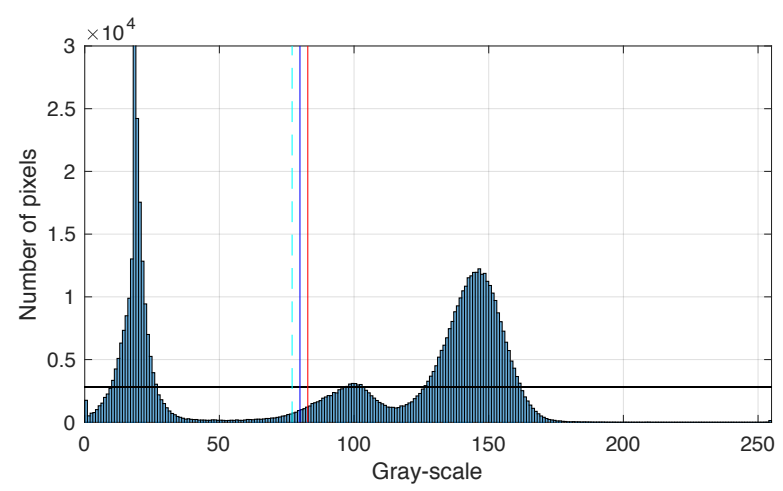

Fig. 4 Histogram of grayscale values

\subsubsection{Otsu's Threshold}

The method described in [16] defines the Otsu threshold. This method tends to minimize the variance of the image intra-class. Using this calculation method, the corresponding threshold, $T_{\text {Otsu }}$, is defined by the cyan vertical dash line in Figure 4 .

While the Otsu method maximizes the separability of gray value classes in bimodal histograms, the Iso50 threshold only considers the exact middle of two material peaks of interest [3]. Because of its integral approach, the Otsu method allows taking into account the global aspect of the histogram and not only the two dominant peaks.

\subsubsection{Weighted Threshold}

The last calculation method proposed is the linear combination of the two previous ones. This allows us to observe the link between the extracted surface and the threshold in the Iso50 to Otsu range. The corresponding threshold is defined according to Equation 1 with $\alpha \in[-1 ; 1]$ :

$$
T_{\alpha}=\frac{1}{2} \cdot\left((1-\alpha) \cdot T_{I_{s o_{50}}}+(1+\alpha) \cdot T_{O t s u}\right)
$$

Using this calculation method, the threshold corresponds to the blue vertical line in figure 4.

\subsection{Surface extraction}

The threshold allows the identification of voxels (or pixels) corresponding to the surface of the object. The calculation of areal texture parameters relies on a mesh of the surface. To extract the mesh from the surface voxels, the marching cube method is used. The algorithm of the marching cubes was developed by Lorensen and Cline [11] for the isosurface extraction as a polygonal mesh from a discrete 
three-dimensional scalar field such as voxels. This algorithm constructs a facetized isosurface by processing the data set in a sequential, cube-by-cube manner. The marching cube algorithm is probably the most popular method for isosurface extraction [14].

Considering eight points of the volumetric data simultaneously, which define a cube, this algorithm determines the polygons to be created corresponding to the isosurface in the considered cube.

An array of the 256 possible polygon configurations is defined by reflection and symmetry of the 15 possible cases (figure 5). For each one of the eight points, an index is affected to the point depending ont its gray value. If the gray value is greater than the given threshold, the index is set to 1 , otherwise the index is set to 0 . The set of indexes is thus compared to the pre-defined array in order to find the similar configuration, thus defining the corresponding polygon (figure 5).

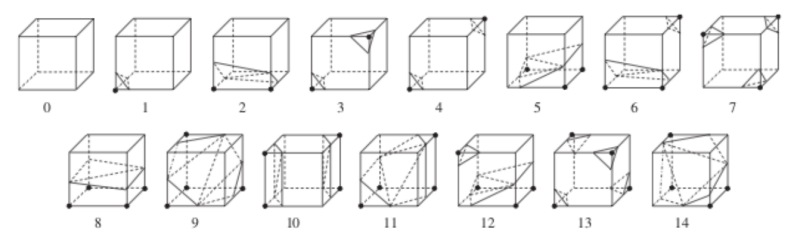

Fig. 5 Description of the marching cube [14] algorithm

This algorithm yields a mesh of the surface defined by the threshold value. The extraction of all the vertices of this mesh results in a point cloud, which is thus exported as a text file to be treated and analyzed using MountainMap. Figure 6 (b) displays the exported point cloud corresponding to the 3D printed part used in [19] and Figure 6(c) displays one slice of the volumetric data of the part.

\subsection{Validation on a reference surface}

In order to assess the process of surface extraction, dimensional measurements of a ceramic calibrated ball (grade 5) are conducted. The characteristics of the artefact are as follows: diameter $=9.525 \mathrm{~mm}$, sphericity accuracy $=0.13 \mu \mathrm{m}$, and diameter accuracy $=+1.25 \mu \mathrm{m} /-1 \mu \mathrm{m}$. The reference surface is measured with a NSI X50 computed tomography (CT) system (Acceleration voltage $180 \mathrm{kV}$ - Filament current $50 \mu \mathrm{A})$. The artefact scans have a voxel size of $21 \times 21 \times 21 \mu \mathrm{m}$. Note that, the measuring noise evaluated to $29 \mu \mathrm{m}$ according to the protocol Qualipso defined in [12] is of the same order as the voxel size. Our method is applied following the steps described in the previous sections using the three different thresholds. Results are summarized in Table 2. Whatever the threshold, the relative deviation between the measured diameter and the reference value is less than $0.1 \%$. This confirms the relevance of the proposed process for dimensional measurements. The next step is now to assess the method for surface topography characterisation. 


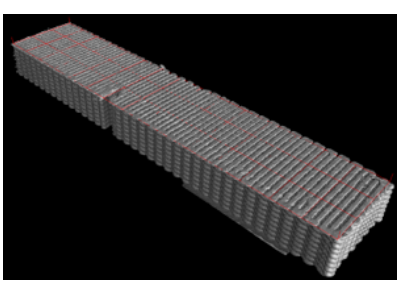

(a) Volumetric data for a printed part in ABS

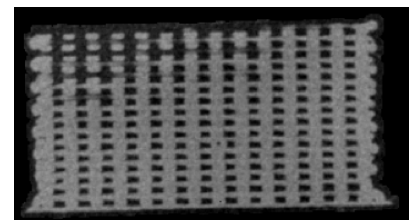

(b) One slice for the printed part in ABS

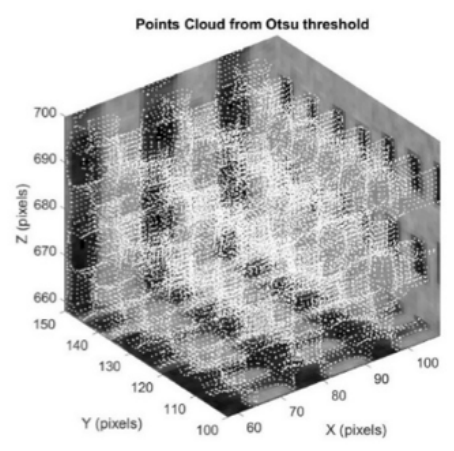

(c) Obtained point cloud

Fig. 6 Example of a 3D printed ABS part

Table 2 Data qualification according to [12]

\begin{tabular}{lllll}
\hline Threshold & Iso $_{50}$ & Otsu & Weighted & Reference \\
\hline Measured Diameter $(\mathrm{mm})$ & 9,5292 & 9.5300 & 9.5296 & 9.525 \\
\hline
\end{tabular}

\section{Experimental protocol}

Surface tests were conducted using two pieces which are classically used in dental prosthetic [10]:

- A block of ceramic $(1 \mathrm{~cm} \times 1 \mathrm{~cm} \times 1.5 \mathrm{~cm})($ Figure $7(\mathrm{a}))$

- A bowl of ceramic $(1 \mathrm{~cm} \times 1 \mathrm{~cm} \times 1 \mathrm{~cm})$ (Figure $7(\mathrm{~b}))$

As presented in [10], samples are made in a nano ceramic composite manufactured by $3 \mathrm{M}$ ESPE. This material has a hardness of $107.3 \mathrm{Hv}$. Both parts are machined according to different cutting conditions as described in [10], and offer an interesting area for roughness measurements. Only one area is analyzed on the block, whereas four areas on the bowl are considered (figure 8).

To assess CT measurements, a measurement is also performed using a classical optical system, which can be set as the reference. The chosen reference system is a focus variation instrument (Alicona InfiniteFocus Standard G4). All the measurements are made with a lateral resolution of $884 \mu \mathrm{m}$ and a vertical resolution of $100 \mathrm{~nm}$. For CT measurements, two tomographs are used: 


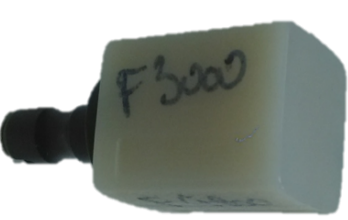

(a) Block specimen

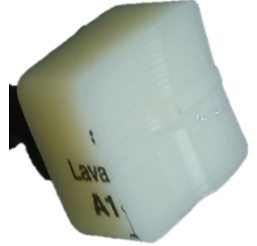

(b) Bowl specimen

Fig. 7 Specimen description

Table 3 Measurement Characteristics

\begin{tabular}{llllll}
\hline \multirow{2}{*}{$\begin{array}{l}\text { System } \\
\text { used }\end{array}$} & \multicolumn{5}{c}{ Voxel Size $\left(\mathrm{mm}^{3}\right)$} \\
\cline { 2 - 6 } & Block & $\mathrm{Z} 1$ & $\mathrm{Z} 2$ & Bowl & $\mathrm{Z} 3$ \\
\hline Nikon & $21^{3}$ & $21^{3}$ & $21^{3}$ & $21^{3}$ & $21^{3}$ \\
NSI & $21^{3}$ & Not used & Not used & Not used & Not used \\
\hline
\end{tabular}

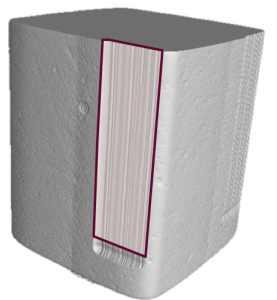

(a) Studied surface on the block

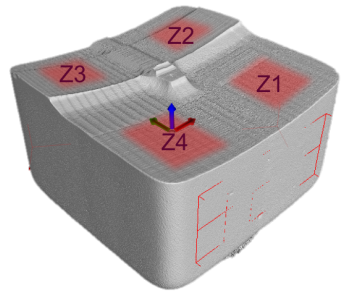

(b) Studied surfaces on the bowl

Fig. 8 Representation of the measured area

- Nikon XT 225 Acceleration voltage $190 k \mathrm{~V}$ - Filament current $80 \mu \mathrm{A}$

- NSI X50 Acceleration voltage $180 \mathrm{kV}$ - Filament current $50 \mu \mathrm{A}$

In our experiment, the NSI system is only used for the block surface. This second system is used to test the consistency of the data obtained after data processing. Measurement characterisitics are given in Table 3.

For each specimen, the process of surface extraction from CT and optical measurements is the same. A $2000 \mu m \times 1950 \mu m$ surface (Figure 9) is extracted in the same area. No filtrering operation is carried out before calculating rougnhess parameters. Results obtained with the optical instrument are displayed in table 4, and corresponding measured surfaces are reported in figures 9 and 10. For ease of reading, only some parameters have been reported in the figure, as the others give similar results. 
Table 4 Areal surface parameters from optical measurements

\begin{tabular}{lllllllll}
\hline & $\begin{array}{l}S_{q} \\
\mu m\end{array}$ & $\begin{array}{l}S_{a} \\
\mu m\end{array}$ & $\begin{array}{l}S_{z} \\
\mu m\end{array}$ & $S_{k u}$ & $S_{s k}$ & $\begin{array}{l}S_{p} \\
\mu m\end{array}$ & $\begin{array}{l}S_{v} \\
\mu m\end{array}$ & $\begin{array}{l}S_{5 v} \\
\mu m\end{array}$ \\
\hline Block & 10,48 & 8,27 & 69,26 & 3,31 & $-0,54$ & 35,02 & 34,25 & 10,72 \\
Z1 & 10,97 & 9,26 & 54,65 & 2,06 & 0,26 & 30,49 & 24,16 & 5,37 \\
Z2 & 5,73 & 4,70 & 40,25 & 2,64 & 0,46 & 25,53 & 14,72 & 5,27 \\
Z3 & 9,87 & 8,28 & 47,59 & 2,14 & 0,38 & 29,08 & 18,51 & 5,09 \\
Z4 & 7,17 & 5,98 & 40,00 & 2,33 & $-0,11$ & 18,18 & 21,82 & 8,30 \\
\hline
\end{tabular}

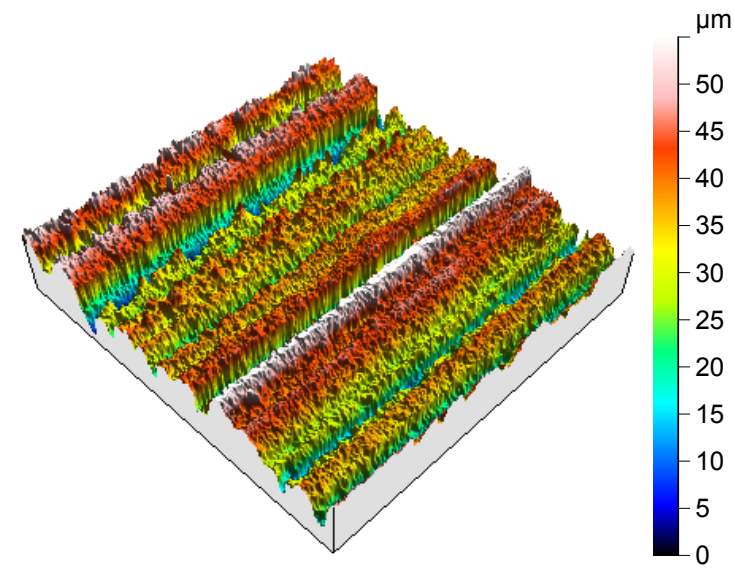

Fig. 9 Optical measurements for the Block

\section{Surface analysis and characterization}

Different studies are then conducted based on the measurements with the two systems. First, the influence of the thresholding is illustrated. In a second case study, measurements obtained using CT are compared to optical measurements. Finally, a more detailed study of measurement deviations allows us to bring out which surface textures are most suitable for tomography measurements.

\subsection{Threshold influence}

To study the influence of the thresholding on surface characterization, only the block is considered for the two CT measuring systems. The different threshold values described in 2.1, $T_{I s_{50}}, T_{O s t u}$ and $T_{W h e i g h t e d}$, are applied to the measured data. For the $T_{W h e i g h t e d}$ threshold, the parameter $\alpha$ is set between -0.5 to 0.5 with an interval (spacing) of 0.25 . Figure 11 illustrates the aspect of the obtained surface for each of the three threshold values after surface extraction. This surface is the basis of areal parameter calculation using MountainMap. Calculated values of roughness parameters are reported in Table 6 .

Results show a slight difference between the two CT systems, which is not significant. The maximum variation is less than $1.3 \mu \mathrm{m}$ for the parameter $S_{q}$, and with $S_{z}$ the difference is less than $4 \%$. In contrast, results clearly highlight 


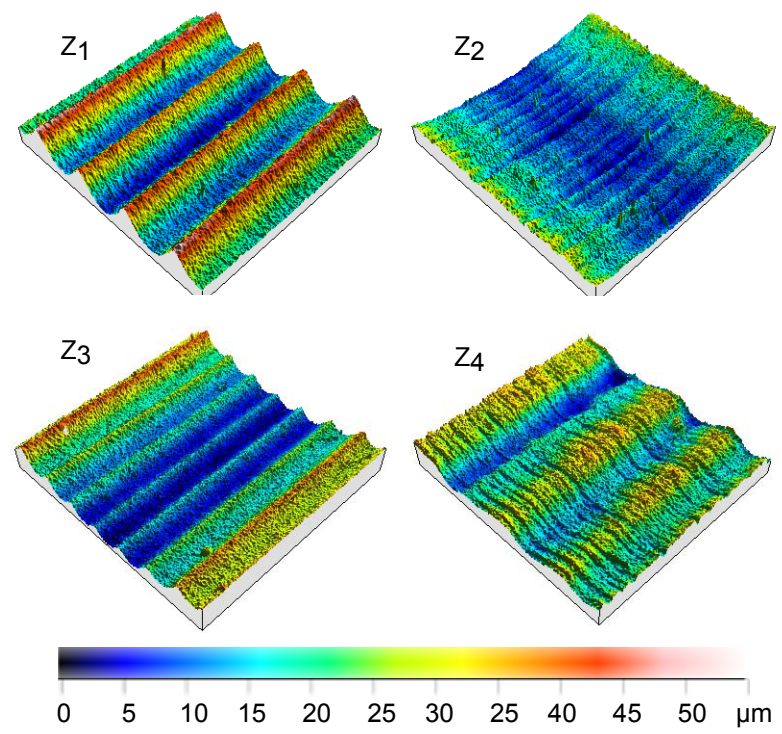

Fig. 10 Optical measurements for the four surfaces of the Bowl

Table 5 Threshold Used

\begin{tabular}{lll}
\hline System Used & $T_{I S O_{50}}$ & $T_{\text {Otsu }}$ \\
\hline Nikon & 80.899 & 79.000 \\
NSI & 72.942 & 70.000 \\
\hline
\end{tabular}

Table 6 Surface areal parameters for the three threshold values

\begin{tabular}{|c|c|c|c|c|c|c|c|c|c|}
\hline & & & $\begin{array}{l}S_{q} \\
\mu m\end{array}$ & $\begin{array}{l}S_{a} \\
\mu m\end{array}$ & $\begin{array}{l}S_{z} \\
\mu m\end{array}$ & $S_{k u}$ & $\begin{array}{l}S_{p} \\
\mu m\end{array}$ & $\begin{array}{l}S_{v} \\
\mu m\end{array}$ & $\begin{array}{l}S_{5 v} \\
\mu m\end{array}$ \\
\hline \multirow{7}{*}{ 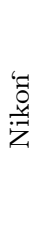 } & & Iso50 & 7.43 & 5.80 & 46.26 & 3.35 & 18.68 & 27.58 & 6.34 \\
\hline & & Otsu & 7.14 & 5.59 & 44.59 & 3.38 & 18.64 & 25.95 & 6.95 \\
\hline & & -0.5 & 7.35 & 5.72 & 46.74 & 3.41 & 18.52 & 28.21 & 6.59 \\
\hline & & -0.25 & 7.27 & 5.65 & 45.15 & 3.43 & 18.02 & 27.13 & 7.09 \\
\hline & $\alpha$ & 0 & 7.29 & 5.71 & 45.56 & 3.35 & 18.33 & 27.23 & 6.94 \\
\hline & & 0.25 & 7.15 & 5.58 & 44.95 & 3.43 & 17.92 & 27.03 & 7.37 \\
\hline & & 0.5 & 7.11 & 5.55 & 44.87 & 3.43 & 18.07 & 26.80 & 6.73 \\
\hline \multirow{7}{*}{$\begin{array}{l}\text { ⿹ } \\
\text { 乙 }\end{array}$} & \multirow{7}{*}{$\alpha$} & Iso50 & 8.42 & 6.65 & 48.11 & 3.39 & 20.71 & 27.39 & 6.70 \\
\hline & & Otsu & 8.34 & 6.53 & 46.33 & 3.42 & 19.09 & 27.24 & 7.21 \\
\hline & & -0.5 & 8.38 & 6.57 & 46.70 & 3.31 & 19.75 & 26.95 & 7.30 \\
\hline & & -0.25 & 8.37 & 6.58 & 47.01 & 3.30 & 20.15 & 26.86 & 7.66 \\
\hline & & 0 & 8.38 & 6.58 & 47.01 & 3.30 & 20.11 & 26.91 & 7.71 \\
\hline & & 0.25 & 8.37 & 6.57 & 47.01 & 3.32 & 20.07 & 26.95 & 7.59 \\
\hline & & 0.5 & 8.37 & 6.56 & 46.06 & 3.33 & 19.31 & 26.75 & 7.10 \\
\hline
\end{tabular}

the influence of the threshold value on parameter evaluation. This influence is not linear, and differs from one parameter to the other. As far as $T_{W h e i g h t e d}$ is concerned, results show that parameter values are very close, and it seems relevant 


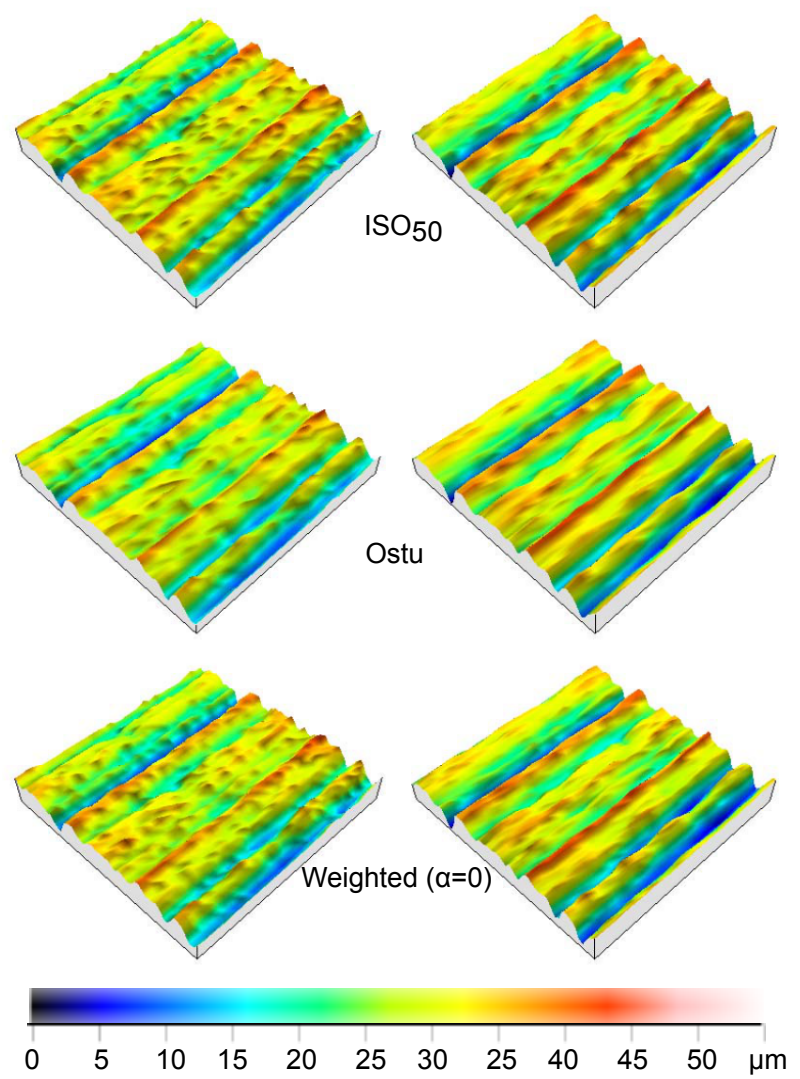

Fig. 11 Aspect of the Block surface measured with Nikkon CT (left column) and NSI CT (right column) for the three thresholds

to only consider the value $\alpha=0$. Finally, only the three thresholds $T_{I_{\text {so }}{ }_{50}}, T_{\text {Ostu }}$ and $T_{W h e i g h t e d}$ with $\alpha=0$ will be used in the next section. Values obtained by both methods (Ostu and Iso $_{50}$ ) strongly depend on the gray level distribution which is itself linked to the nature of the material. In our case the difference between both values is small, but it must be noticed that it could be larger with another type of material.

\subsection{Surface areal Analysis}

This section only focuses on results obtained from measurements made with the Nikon CT. Results concern the block (A table 9)and the four zones of the bowl (A table 10, 11, 12, 13). Only six amplitude parameters are studied according to [6], and results obtained using the optical measuring system are taken as the reference.

Results for $S_{a}$ and $S_{q}$ are quite good for Z1, Z2 and Z3 with about $10 \%$ of error, which is satisfying considering the rather large voxel size $(20 \mu \mathrm{m})$. On the contrary, 
results are unsatisfactory for the block and Z4. For most specimens, parameter $S_{z}$ and $S_{p}$ are not properly identified. These parameters are more difficult to identify correctly than $S_{a}$ and $S_{q}$, which are averaged parameters. Figure 12 clearly highlights that the low resolution of the system greatly reduces the information that can be obtained on the texture of the surface, which is reflected in the errors observed in the parameters. Nevertheless, results show that depending on the nature of the surface texture, errors are more or less significant. The following section is dedicated to the analysis of this influence in order to highlight which typology of surface is better to evaluate surface roughness parameters based on CT measurements.
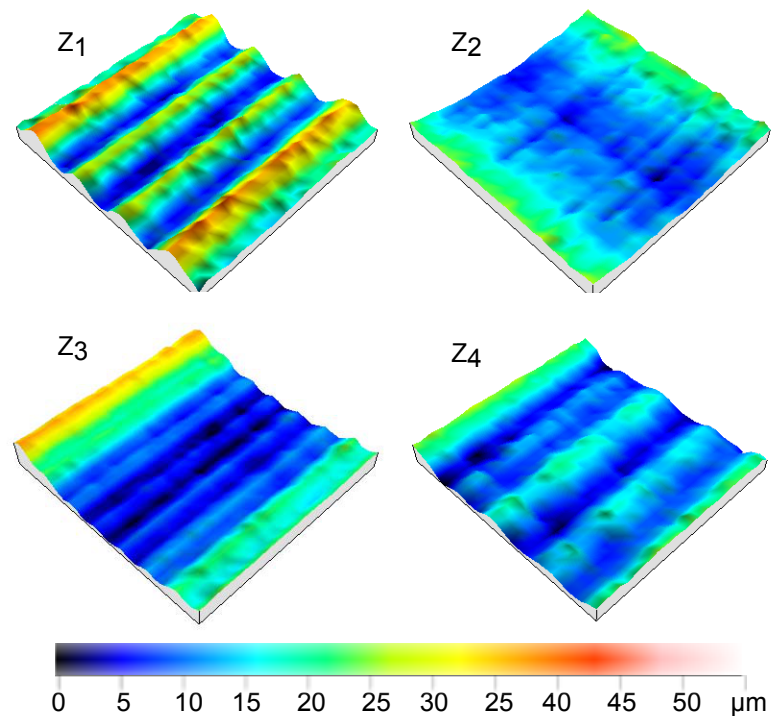

Fig. 12 Representation of the Nikon measurement for the four surfaces of the bowl according to Otsu's threshold

\subsection{Discussion}

Results reported in Section 4.2 brought out that the determination of surface roughness parameters may be relevant for some surface topographies and not for others. The purpose of this section is to highlight the texture characteristics that may influence these results.

First, to check the diversity of the specimens used for this study, a KruskallWallis test is performed. Data from Table 4 are used to perform statistical analysis of variance. From the calculated p-value (Table 7 ), it could be assumed that the specimens used could be considered as significantly different.

Surface roughness parameters determined with volumetric data from CT and the optical system (A) show different deviations depending on the studied sur- 
Table 7 p-values from Kruskal-Wallis test

\begin{tabular}{ll}
\hline Parameter & p-value \\
\hline$S_{s k}$ & 0.009 \\
$S_{5 v}$ & 0.011 \\
$S_{k u}$ & 0.009 \\
\hline
\end{tabular}

Table 8 Correlation coefficient

\begin{tabular}{|c|c|c|c|}
\hline Data & $S_{s k}$ & $S_{5 v}$ & $S_{k u}$ \\
\hline $\begin{array}{l}\text { Relative mean devia- } \\
\text { tion for } S_{a}\end{array}$ & 0.961 & -0.983 & \\
\hline $\begin{array}{l}\text { Relative mean devia- } \\
\text { tion for } S_{q} \\
\text { Relative mean devia- } \\
\text { tion for } S_{z}\end{array}$ & 0.951 & -0.983 & -0.956 \\
\hline
\end{tabular}

face. In order to link these deviations to the texture, an analysis was carried out on the correlation of these deviations with the surface roughness parameters determined with the optical system which serves as reference (Table 4). Table 8 displays results of the strongest correlations obtained. Plots for these data (figure 13) clearly show a high error dependency for $S_{q}$ deviation with $S_{s k}$ and $S_{5 v}$, as well as $S_{z}$ deviation with $S_{k u}$. According to Figure 13(b), it appears that the more negative the skewness is, the better the results according to $S_{q}$. The more nonsymmetrically distributed the heights of the surface are, and the smaller they are compared to the average, the better the determination of $S_{q}$. The results are the same for $S_{a}$. In addition, errors on the parameter $S_{q}$ (Figure 13(a)) are reduced for surface topographies whose main valleys have a lower depth. For the study of the measurement quality of $S_{z}$ according to the kurtosis evolution (Figure 13(c)), it is preferable that $S_{k u}$ be small, which corresponds to a surface with the most uniformly distributed height possible.

\section{Conclusion}

The objective of the works presented in this paper is to study the feasibility of measuring surface roughness by X-ray computed tomography (CT). This feasibility is studied more particularly in the case of a low-resolution measurement, which happens when both the overall geometry and the local topography of the part are measured without extracting a specimen. Thus, the relative positions of the part to the source and the target prevent refinement of this resolution. Given the cost of using such a machine, the possibility of measuring both the shape and the topography of the part at the same time makes sense with the view of further industrialization of this measurement process. To this end, a surface extraction process from volumetric data using the marching cube algorithm was developed. Then, this methodology was validated on a form defect artifact.

The choice of the threshold value is an important parameter for the quality of the extracted data. Its determination depends on both global and local grayscale histograms. In order to validate the choice of a pre-selection method, different 


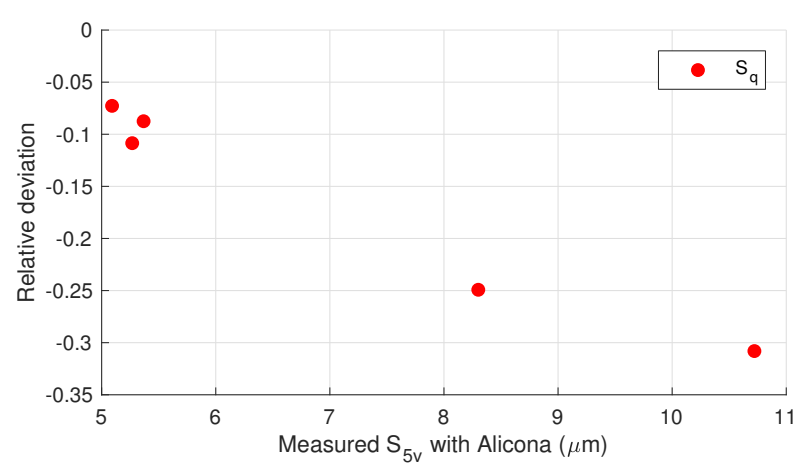

(a) Relation for $S_{5 v}$

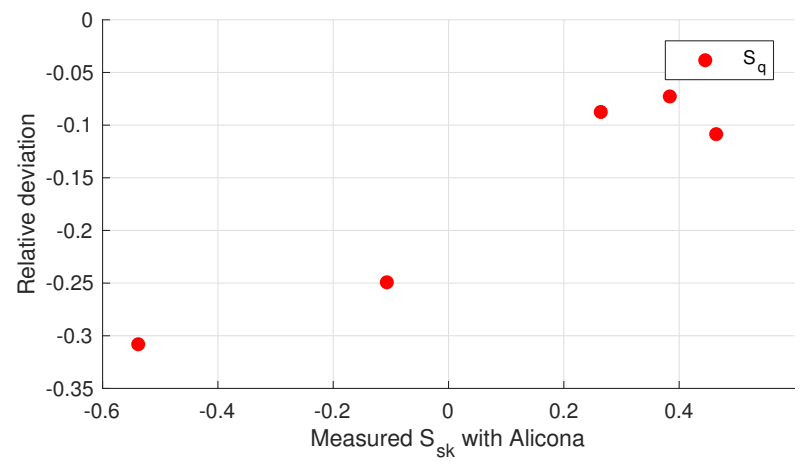

(b) Relation for $S_{s k}$

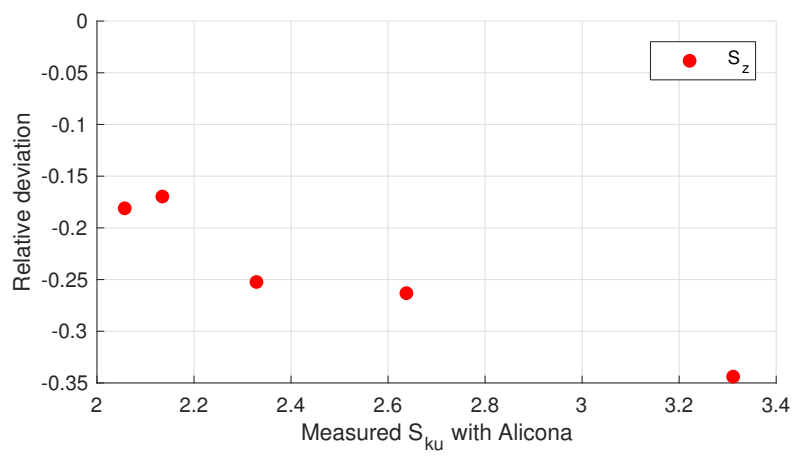

(c) Relation for $S_{k u}$

Fig. 13 Representation of the data correlation

thresholds were tested. Results showed that these thresholds have little influence on the determination of surface roughness parameters, whatever the CT system used.

Finally, the study carried out on four different specimens highlighted differences in the estimation of surface roughness parameters between tomography measurements and classical optical measurements. These deviations clearly depend on 
the initial topography of the studied surface. A more advanced analysis of the correlation between these deviations and the topography revealed which surface typologies are more appropriate for CT measurements with poor resolution.

This work will now be continued in order to integrate measurement uncertainties and confirm announced results. It is also planned to change the shape of the samples to internal shapes, that more correspond to lattice structures. One of the ways to improve the determination of surface topography by CT measurements is to carry out a study on a finer subpixel scale than that currently used.

\section{Acknowledgements}

Nikon Measurement which provided a part of CT measurements. CT measurements at ENS Paris-Saclay has been financially supported by the French "Agence Nationale de la Recherche," through the "Investissements d'avenir" program (ANR10- EQPX-37 MATMECA Grant).

\section{References}

1. Valentina Aloisi, Simone Carmignato Influence of surface roughness on X-ray computed tomography dimensional measurements of additive manufactured parts Case Studies in Nondestructive Testing and Evaluation 6, 104-110 (2016)

2. C. Beyer, D. Figueroa, Design and Analysis of Lattice Structures for Additive Manufacturing, ASME Journal of Manufacturing Science and Engineering, 138, 121014, (2016).

3. Simone Carmignato, Wim Dewulf, Richard Leach, Industrial X-Ray Computed Tomography, Springer International Publishing (2018).

4. Chiffre, L. D., Carmignato, S., Kruth, J.-P., Schmitt, R., and Weckenmann, A. . Industrial applications of computed tomography. CIRP Annals - Manufacturing Technology, 63, 655 $677,(2014)$.

5. Melvin Diaza, Kwang Yeom Kimb, Sun Yeomb, Li Zhuangb, Sehyeok Parkc, Ki-Bok Minc Surface roughness characterization of open and closed rock joints in deep cores using Xray computed tomography International Journal of Rock Mechanics \& Mining Sciences 98, 1019 (2017)

6. ISO 25178-2. "Geometrical Product Specifications (GPS) Surface Texture Areal Part 2: Terms, Definitions and Surface Texture Parameters," International Organization for Standardization, 2012.

7. R. Jimènez, C. Comps and J. A. Yagüe, "An Optimized Segmentation Algorithm for the Surface Extraction in Computed Tomography for Metrology Applications," Procedia Engineering, 132, 804-810 (2015)

8. Kerckhofs G, Pyka G, Moesen M, Van Bael S, Schrooten J, Wevers M. High-resolution microfocus X-ray computed tomography for 3D surface roughness measurements of additive manufactured porous materials. Adv Eng Mater 15:1538, (2013).

9. Tomasz Kowaluk, Adam Wozniak, A new threshold selection method for X-ray computed tomography for dimensional metrology, Precision Engineering, 50, 449-454, (2017)

10. N. Lebon, L. Tapie, E. Vennat and B. Mawussi, "Influence of CAD/CAM tool and material on tool wear and roughness of dental prostheses after milling", The Journal of Prosthetic Dentistry, 114, 236-247. (2015)

11. W. E. Lorensen and H. E. Cline, "Marching cubes: A high resolution 3D surface construction algorithm," in ACM siggraph computer graphics, (1987)

12. Mehdi-Souzani, C., Quinsat, Y., Lartigue, C., Bourdet, P., A knowledge database of qualified digitizing systems for the selection of the best system according to the application. CIRP Journal of Manufacturing Science and Technology, 13, 15-23, (2016)

13. Giovanni Moronia, Stefano Petro Impact of the Threshold on the Performance Verification of Computerized Tomography Scanners 14th CIRP Conference on Computer Aided Tolerancing (CAT) Procedia CIRP 43, 345 350(2016) 
14. Timothy S. Newman, Hong Yi A survey of the marching cubes algorithm Computers \& Graphics 30, 854-879,(2006)

15. Andreas F. Obrist, Alexander Flisch, Juergen Hofmann Point cloud reconstruction with sub-pixel accuracy by slice-adaptive thresholding of X-ray computed tomography images NDT\&E International 37, 373-380 (2004)

16. N. Otsu, A threshold selection method from gray-level histograms, IEEE transactions on systems, man, and cybernetics, 9, 62-66 (1979)

17. Pyka G, Kerckhofs G, Papantoniou I, Speirs M, Schrooten J, Wevers M. Surface roughness and morphology customization of additive manufactured open porous Ti6Al4V structures. Materials 6:473757, (2013).

18. Y. Quinsat, C. Lartigue, C. A. Brown and L. Hattali, "Multi-scale surface characterization in additive manufacturing using CT," in Lecture Notes in Mechanical Engineering, Springer International Publishing, 271-280, (2016).

19. Y. Quinsat, C. Lartigue, C. Brown, L. Hattali, Characterization of surface topography of 3D printed parts by multi-scale analysis, International Journal on Interactive Design and Manufacturing, DOI: 10.1007/s12008-017-0433-9, (2017)

20. N Senin, A Thompson and R K Leach, Characterisation of the topography of metal additive surface features with different measurement technologies, Measurement Science and Technology, Volume 27, Number 28, 095003, (2017)

21. A Thompson, I Maskery and R K Leach, X-ray computed tomography for additive manufacturing: a review, Measurement Science and Technology, Volume 27, Number 7, (2016)

22. A. Thompson, L. Krner, N. Senin, S. Lawes, I. Maskery and R. Leach, "Measurement of internal surfaces of additively manufactured parts by X-ray computed tomography," ICT 2017 (2017)

23. A. Townsend, N. Senin, L. Blunt, R. K. Leach and J. S. Taylor, "Surface texture metrology for metal additive manufacturing: a review," Precision Engineering, 46, 34-47, (2016).

24. Marta Torral, Roberto Jimenez, Jose A. Yague-Fabra, Sinue Ontiveros, Guido Tosello, Comparison of surface extraction techniques performance in computed tomography for 3D complex micro-geometry dimensional measurements, The International Journal of Advanced Manufacturing Technology, https://doi.org/10.1007/s00170-018-1950-9, 2018.

25. A. Townsend, L. Pagani, P. Scott and L. Blunt, "Areal surface texture data extraction from X-ray computed tomography reconstructions of metal additively manufactured parts," Precision Engineering, 48, 254-264. (2017)

26. A. Townsend, L. Pagani, L. Blunt, P. J. Scott and X. Jiang, "Factors affecting the accuracy of areal surface texture data extraction from X-ray CT," CIRP Annals - Manufacturing Technology, 66, 547-550, (2017).

27. Albert Weckenmann, Philipp Kramer, Application of Computed Tomography in Manufacturing Metrology, Technisches Messen, Volume 76, Issue 7-8 (Jul 2009).

\section{A Appendix A: Measurement results}


Table 9 Surface areal parameters for Block1

\begin{tabular}{lllll}
\hline Parameter & Threshold & Nikon & Alicona & $\begin{array}{l}\text { Relative } \\
\text { Mean } \\
\text { deviation }\end{array}$ \\
\hline \multirow{3}{*}{$S_{q} \mu m$} & Iso50 & 7,43 & & \\
& Ostu & 7,14 & 10,48 & $-30,81 \%$ \\
& Weighted & 7,29 & & \\
\hline \multirow{5}{*}{$S_{a} \mu m$} & Iso50 & 5,80 & & \\
& Ostu & 5,59 & 8,27 & $-31,61 \%$ \\
& Weighted & 5,71 & & \\
\hline \multirow{5}{*}{$S_{z \mu m}$} & Iso50 & 46,26 & & \\
& Ostu & 44,59 & 69,26 & $-34,39 \%$ \\
& Weighted & 45,56 & & \\
\hline \multirow{5}{*}{$S_{k u}$} & Iso50 & 3,35 & & \\
& Ostu & 3,38 & 3,31 & $2,64 \%$ \\
& Weighted & 3,35 & & \\
\hline \multirow{5}{*}{$S_{p} \mu m$} & Iso50 & 18,68 & & \\
& Ostu & 18,64 & 35,01 & $-47,71 \%$ \\
& Weighted & 18,33 & & \\
\hline \multirow{5}{*}{$S_{v} \mu m$} & Iso 50 & 27.58 & & \\
& Ostu & 25,95 & 34,25 & $-20,77 \%$ \\
& Weighted & 27,23 & & \\
\hline \multirow{5}{*}{$S_{5 v} \mu m$} & Iso50 & 6.34 & & \\
& Ostu & 6.95 & 10.72 & $-36.02 \%$ \\
& Weighted & 7.09 & & \\
\hline
\end{tabular}

Table 10 Surface areal parameters for Zone 1

\begin{tabular}{lllll}
\hline Parameter & Threshold & Nikon & Alicona & $\begin{array}{l}\text { Relative } \\
\text { Mean } \\
\text { deviation }\end{array}$ \\
\hline \multirow{3}{*}{$S_{q} \mu m$} & Iso50 & 10.01 & & \\
& Ostu & 10,00 & 10,97 & $-8,75 \%$ \\
& Weighted & 10,01 & & \\
\hline \multirow{5}{*}{$S_{a} \mu m$} & Iso50 & 8,36 & & \\
& Ostu & 8,34 & 9,26 & $-9,83 \%$ \\
& Weighted & 8,36 & & \\
\hline \multirow{5}{*}{$S_{z} \mu m$} & Iso50 & 19,61 & & \\
& Ostu & 20,09 & 24,16 & $-18,11 \%$ \\
& Weighted & 19,85 & & \\
\hline \multirow{5}{*}{$S_{k u}$} & Iso50 & 2,17 & & \\
& Ostu & 2,17 & 2,06 & $5,54 \%$ \\
& Weighted & 2,17 & & \\
\hline \multirow{5}{*}{$S_{p} \mu m$} & Iso50 & 25,09 & & \\
& Ostu & 24,70 & 30,49 & $-18,32 \%$ \\
& Weighted & 24,92 & & \\
\hline \multirow{5}{*}{$S_{v} \mu m$} & Iso50 & 19,61 & & \\
& Ostu & 20,09 & 24,16 & $-17,84 \%$ \\
& Weighted & 19,85 & & \\
\hline \multirow{3}{*}{$S_{5 v} \mu m$} & Iso50 & 5.78 & & \\
& Ostu & 5.81 & 5.37 & $7.65 \%$ \\
& Weighted & 5.75 & & \\
\hline
\end{tabular}


Table 11 Surface areal parameters for Zone 2

\begin{tabular}{|c|c|c|c|c|}
\hline Parameter & Threshold & Nikon & Alicona & $\begin{array}{l}\text { Relative } \\
\text { Mean } \\
\text { deviation }\end{array}$ \\
\hline$S_{q} \mu m$ & $\begin{array}{l}\text { Iso50 } \\
\text { Ostu } \\
\text { Weighted }\end{array}$ & $\begin{array}{l}5,10 \\
5,10 \\
5,11 \\
\end{array}$ & 5,73 & $-10,85 \%$ \\
\hline$S_{a} \mu m$ & $\begin{array}{l}\text { Iso50 } \\
\text { Ostu } \\
\text { Weighted }\end{array}$ & $\begin{array}{l}4,22 \\
4,22 \\
4,23 \\
\end{array}$ & 4,70 & $-10,05 \%$ \\
\hline$S_{z} \mu m$ & $\begin{array}{l}\text { Iso50 } \\
\text { Ostu } \\
\text { Weighted }\end{array}$ & $\begin{array}{l}29,82 \\
29,27 \\
29,88 \\
\end{array}$ & 40,25 & $-26,32 \%$ \\
\hline$S_{k u}$ & $\begin{array}{l}\text { Iso50 } \\
\text { Ostu } \\
\text { Weighted }\end{array}$ & $\begin{array}{l}2,41 \\
2,41 \\
2,41 \\
\end{array}$ & 2,64 & $-8,61 \%$ \\
\hline$S_{p} \mu m$ & $\begin{array}{l}\text { Iso50 } \\
\text { Ostu } \\
\text { Weighted }\end{array}$ & $\begin{array}{l}15,67 \\
15,11 \\
15,55\end{array}$ & 25,53 & $-39,50 \%$ \\
\hline$S_{v} \mu m$ & $\begin{array}{l}\text { Iso50 } \\
\text { Ostu } \\
\text { Weighted }\end{array}$ & $\begin{array}{l}14,15 \\
14,16 \\
14,33\end{array}$ & 14,72 & $-3,45 \%$ \\
\hline$S_{5 v} \mu m$ & $\begin{array}{l}\text { Iso50 } \\
\text { Ostu } \\
\text { Weighted }\end{array}$ & $\begin{array}{l}4.59 \\
4.63 \\
5.01\end{array}$ & 5.27 & $-9.94 \%$ \\
\hline
\end{tabular}

Table 12 Surface areal parameters for Zone 3

\begin{tabular}{lllll}
\hline Parameter & Threshold & Nikon & Alicona & $\begin{array}{l}\text { Relative } \\
\text { Mean } \\
\text { deviation }\end{array}$ \\
\hline \multirow{3}{*}{$S_{q} \mu m$} & Iso50 & 9,13 & & \\
& Ostu & 9,19 & 9,85 & $-7,27 \%$ \\
& Weighted & 9,10 & & \\
\hline \multirow{5}{*}{$S_{a} \mu m$} & Iso50 & 7,62 & & \\
& Ostu & 7,63 & 8,28 & $-8,02 \%$ \\
& Weighted & 7,59 & & \\
$S_{z} \mu m$ & Iso50 & 39,27 & & \\
& Ostu & 39,63 & 47,59 & $-16,97 \%$ \\
& Weighted & 39,64 & & \\
\hline \multirow{5}{*}{$S_{k u}$} & Iso50 & 2,90 & & \\
& Ostu & 3,03 & 2,13 & $39,44 \%$ \\
& Weighted & 3,00 & & \\
\hline \multirow{5}{*}{$S_{p} \mu m$} & Iso50 & 25,33 & & \\
& Ostu & 25,73 & 29,08 & $-11,90 \%$ \\
& Weighted & 25,80 & & \\
\hline \multirow{5}{*}{$S_{v} \mu m$} & Iso50 & 13,94 & & \\
& Ostu & 13,89 & 18,50 & $-24,93 \%$ \\
\hline \multirow{5}{*}{$S_{5 v} \mu m$} & Weighted & 13,84 & & \\
\hline & Iso50 & 3.44 & & \\
\hline & Weighted & 3.45 & & \\
\hline
\end{tabular}


Table 13 Surface areal parameters for Zone 4

\begin{tabular}{|c|c|c|c|c|}
\hline Parameter & Threshold & Nikon & Alicona & $\begin{array}{l}\text { Relative } \\
\text { Mean } \\
\text { deviation }\end{array}$ \\
\hline$S_{q} \mu m$ & $\begin{array}{l}\text { Iso } 50 \\
\text { Ostu } \\
\text { Weighted }\end{array}$ & $\begin{array}{l}5,41 \\
5,34 \\
5,41 \\
\end{array}$ & 7,17 & $-30,81 \%$ \\
\hline$S_{a} \mu m$ & $\begin{array}{l}\text { Iso50 } \\
\text { Ostu } \\
\text { Weighted }\end{array}$ & $\begin{array}{l}4,42 \\
4,37 \\
4,42\end{array}$ & 5,98 & $-31,61 \%$ \\
\hline$S_{z} \mu m$ & $\begin{array}{l}\text { Iso50 } \\
\text { Ostu } \\
\text { Weighted }\end{array}$ & $\begin{array}{l}30,63 \\
28,60 \\
30,49 \\
\end{array}$ & 40,00 & $-34,39 \%$ \\
\hline$S_{k u}$ & $\begin{array}{l}\text { Iso } 50 \\
\text { Ostu } \\
\text { Weighted }\end{array}$ & $\begin{array}{l}2,65 \\
2,57 \\
2,65\end{array}$ & 2,33 & $2,64 \%$ \\
\hline$S_{p} \mu m$ & $\begin{array}{l}\text { Iso50 } \\
\text { Ostu } \\
\text { Weighted }\end{array}$ & $\begin{array}{l}17,91 \\
16,31 \\
17,92 \\
\end{array}$ & 18,18 & $-47,71 \%$ \\
\hline$S_{v} \mu m$ & $\begin{array}{l}\text { Iso50 } \\
\text { Ostu } \\
\text { Weighted }\end{array}$ & $\begin{array}{l}12,72 \\
12,29 \\
12,57\end{array}$ & 21,82 & $-20,77 \%$ \\
\hline$S_{5 v} \mu m$ & $\begin{array}{l}\text { Iso50 } \\
\text { Ostu } \\
\text { Weighted }\end{array}$ & $\begin{array}{l}3.96 \\
4.90 \\
3.99\end{array}$ & 8.30 & $-48.41 \%$ \\
\hline
\end{tabular}

